\title{
WEIGHTED HARDY AND PÓLYA-KNOPP INEQUALITIES WITH VARIABLE LIMITS
}

\author{
L. Nikolova, L.-E. Persson, E. Ushakova and A. Wedestig
}

Abstract. A new scale of characterizations for the weighted Hardy inequality with variable limits is proved for the case $1<p \leqslant q<\infty$. A corresponding scale of characterizations for the (limit) weighted Pólya-Knopp inequality is also derived and discussed.

Mathematics subject classification (2000): 26D15, 26D20. limits.

Key words and phrases: inequalities, Hardy type inequalities, Pólya-Knopp type inequalities, variable

\section{REFERENCES}

[1] H. P. HeInIG, G. SinNAmon, Mapping properties of integral averaging operators, Studia Math., 129, (2) (1998), 157-177.

[2] B. OPIC, P. GuRKA, Weighted inequalities for geometric means, Proc. Amer. Math. Soc., 120, (3) (1994), $771-779$.

[3] A. Kufner, L.-E. Persson, Weighted inequalities of Hardy type, World Scientific Publishing Co., Inc., River Edge, NJ, 2003. xviii+357 pp. ISBN: 981-238-195-3.

[4] L.-E. PERSSON, D. PROKHOROV, Integral inequalities for some weighted geometric mean operator with variable limits, Arch. Inequal. Appl., 2,(4) (2004), 475-482.

[5] A. WedESTIG, Weighted inequalities of Hardy-type and their limiting inequalities, $\mathrm{PhD}$ Thesis 2003:17, Department of Mathematics, Luleå University of Technology, 2003. 